\title{
Engagement en ciudades inteligentes. Diseño de un marco de análisis teórico y aplicado para la participación ciudadana
}

\section{Engagement in smartcities. Design of a framework of Theoretical analysis applied to citizen participation}

\author{
María E. Cortés-Cediel \\ Universidad Complutense de Madrid \\ mcorte04@ucm.es
}

\section{NOTA BIOGRÁFICA}

Estudiante de Doctorado en Ciencias Políticas y de la Administración y Relaciones Internacionales. Facultad de Ciencias Políticas y Sociología. Universidad Complutense de Madrid.

\author{
Olga Gil \\ Universidad Complutense de Madrid \\ olgagil@ucm.es
}

\section{NOTA BIOGRÁFICA}

Olga Gil es doctora en Ciencias Políticas y en Sociología (Instituto Universitario Europeo, Florencia, Italia, 2000). Master of Arts en Ciencias Políticas (Universidad de Carolina del Norte en Chapel Hill, 1994), Diploma de Posgrado en Desarrollo y América Latina (UNC y Universidad de Duke, 1994). Licenciada en Periodismo, en Ciencias Políticas y en Sociología (UCM, Madrid). Profesora acreditada con intereses en políticas públicas, gobierno local, tecnología, regulación y transformación digital en las ciudades. Ejerce como profesora asociada en el Departamento de Historia Política, Teorías y Geografía de la Universidad Complutense de Madrid e imparte cursos de posgrado en universidades nacionales e internacionales. Miembro del proyecto de la UE CITADEL (2016-2020): Empoderamiento de los ciudadanos para transformar las administraciones públicas europeas, en el marco del Programa Marco H2020 (call H2020SC6-CULT-COOP-2017-2017, Propuesta 726755). Miembro del Smart Cities and Smart Governments Research-Practice Consortium con sede en el Centro de Tecnología en el Gobierno de la Universidad de Albany. Ha trabajado para la ONU en sus sede de Nueva York y para empresas del IBEX35.

\section{RESUMEN}

El engagement puede definirse como un estado psicológico en el que el individuo centra toda su atención y disfruta de la actividad que está realizando (Salanova y Schaufeli, 2004). La literatura resalta la importancia de la búsqueda de ese estado psicológico por parte de gobiernos y administraciones en el ámbito de la participación ciudadana. Sin embargo, hasta donde llega nuestro conocimiento, las claves previstas en la literatura sobre cómo puede fomentarse el engagement en participación ciudadana no han sido estudiadas en profundidad. Abordando este hecho, este artículo tiene como objetivo elaborar un marco teórico del engagement del ciudadano en participación ciudadana dentro de las ciudades inteligentes. Para ello, en primer lugar, se ofrece una definición del concepto y se identifican una serie de atributos que lo caracterizan. Además se presenta una revisión de la literatura sobre herramientas de participación 
en ciudades inteligentes que pueden estar relacionadas con determinados atributos del engagement. Los resultados principales del estudio son la elaboración de un marco teórico con aplicaciones prácticas que integra atributos característicos del engagement en el campo de la participación ciudadana, y la identificación de aspectos que pueden contener las herramientas de participación para incrementar los niveles de engagement de los ciudadanos.

\title{
PALABRAS CLAVE
}

Engagement; digital citizen engagement; ciudad inteligente; participación ciudadana.

\begin{abstract}
Engagement can be defined as a psychological state in which an individual focuses all her attention and enjoys the activity she is doing. The literature highlights the importance of improving this in citizen participation by governments and administrations. However, to the best of our knowledge, the literature does not offer clues about how engagement in citizen participation can be improved. This paper aims to develop a theoretical framework of citizen engagement for citizen participation in the context of smart cities. To this end, we first provide a definition of engagement, and describe some of its main characteristics. Next, we present a review of the literature on citizen participation tools in smart cities, analyzing if such tools do influence engagement attributes. The main outcomes of the study are the elaboration of a theoretical framework that integrates characteristic attributes of engagement in the field of citizen participation, and the identification of participation tools analyzing whether they can be designed to increase citizen engagement levels.
\end{abstract}

\section{KEYWORDS}

Engagement; digital citizen engagement; smart city; citizen participation.

\section{SUMARIO}

1. INTRODUCCIÓN. 2. CITIZEN ENGAGEMENT: DEFINICIÓN Y PROPUESTA DE MARCO TEÓRICO. ATRIBUTOS EMOCIONALES. ATRIBUTOS SENSORIALES. 3. FASES DEL ENGAGMENT. 3.1. PUNTO DE PARTIDA O INTRODUCCIÓN DEL ENGAGEMENT. 3.2. MANTENIMIENTO DE ENGAGEMENT. 3.3. DISENGAGEMENT. 3.4. REENGAGEMENT. 3.5. NO ENGAGEMENT. 4. PARTICIPACIÓN CIUDADANA EN LAS CIUDADES INTELIGENTES: REVISIÓN DE HERRAMIENTAS DE PARTICIPACIÓN. HERRAMIENTAS FÍSICAS. HERRAMIENTAS VIRTUALES. 5. PROPUESTA DE HERRAMIENTAS PARA CADA NIVEL DE PARTICIPACIÓN CIUDADANA. NIVEL DE INFORMACIÓN. NIVEL DE CONSULTA. NIVEL DE DELEGACIÓN DE PODER. 6. IMPACTO DE LAS HERRAMIENTAS DE PARTICIPACIÓN SOBRE LOS ATRIBUTOS DEL ENGAGEMENT. 7. MEDICIÓN DE ATRIBUTOS DE ENGAGEMENT EN HERRAMIENTAS DE PARTICIPACIÓN. 8. CONCLUSIONES Y TRABAJO FUTURO. REFERENCIAS BIBLIOGRÁFICAS.

\section{INTRODUCCIÓN}

En el año 2050 aproximadamente un 70\% de la población mundial vivirá en ciudades (United Nations, 2008). La importancia de las áreas urbanas como fenómeno global ha desembocado en la difusión de megaciudades de más de 20 millones de personas en Asia y Latinoamérica (Albino et al., 2015). En este escenario lleno de retos hacia los gestores de las ciudades, han proliferado los planes de «smart cities» o ciudades inteligentes, con el objetivo de mitigar los problemas en el ámbito urbano y alcanzar un desarrollo de las ciudades más sostenible (Alawadhi et al., 2012).

En la década de 1990 se observó el potencial de aplicar las Tecnologías de la Información y la Comunicación (TIC) dentro de las ciudades (Albino et al., 2015) para mejorar su desarrollo y sostenibilidad. Este hecho ha ido evolucionando hasta transformar la idea de ciudad inteligente en un concepto transversal aplicable a diferentes ámbitos que ha supuesto una serie de transformaciones económicas, políticas, sociales y culturales (Gil et al., 2016).

Anttiroiko (2016) define ciudad inteligente como «aquel modelo de desarrollo urbano multidimensional con unas TIC avanzadas que permiten potenciar la inteligencia colectiva y la capacidad de incrementar la competitividad y efectividad en las ciudades, y la mejora de la calidad de vida en la comunidad urbana». Se 
observa, por tanto, el potencial de la ciudad inteligente desde distintos enfoques aprovechando sus recursos e infraestructuras en aplicaciones de diversa índole. Según los modelos más citados, los enfoques principales por los que puede considerarse que una ciudad sea inteligente son: "Smart Economy», "Smart Mobility», "Smart Environment», "Smart People», "Smart Living» y "Smart Governance» (Chourabi et al., 2011; European Parliament, 2014; Giffinger et al., 2007). «Smart governance» es el enfoque centrado principalmente en involucrar al ciudadano en la toma de decisiones dentro de la vida local, entre otras cuestiones (Chourabi et al., 2011; Giffinger et al., 2007; Rodríguez-Bolívar y Meijer, 2015).

En las últimas décadas han descendido los niveles de confianza por parte de la ciudadanía hacia las instituciones públicas y al sistema que las sustenta (Ganuza Fernández, 2008; Subirats, 2013). Por ello, la administración pública se ha abierto al ciudadano a través de políticas de rendición de cuentas, transparencia e integración; para volver a ganarse su confianza y recuperar la legitimidad (Díaz, 2017; Gil et al. 2016). Una de las claves para la renovación de esta legitimidad democrática es la participación ciudadana. La búsqueda de un ciudadano más activo e involucrado en su entorno político ha llevado a los gestores públicos a dotar al ciudadano de herramientas necesarias para la participación, introduciendo conceptos nuevos como el engagement. El engagement en el ámbito de la participación ciudadana se refiere al proceso de informar a los ciudadanos, animarlos y motivarlos para fomentar su participación en la toma de decisiones en todas sus fases, desde el diseño hasta su implementación (Pham y Linehan, 2016; Reddel y Woolcock, 2004). Sin embargo, cuestiones sobre cómo conseguir esto y sobre cómo aplicarlo aún son áreas muy poco estudiadas y desarrolladas dentro de la literatura sobre la materia (Bull y Azennoud 2016).

En este artículo se aborda el concepto de engagement en participación ciudadana mediante una propuesta de marco teórico detallado. En él se propone una definición de engagement y se identifica una serie de características o atributos que lo conforman. Además, se realiza una revisión de la literatura sobre herramientas de participación ciudadana existentes en las ciudades inteligentes. El objetivo ha sido identificar aquellas herramientas que podrían potenciar ciertos atributos del engagement. En la literatura se suele referirse al hecho de involucrar al ciudadano a través de la tecnología como «digital citizen engagement» (Group, W. B., 2016). Sin embargo, en este trabajo se propone un marco teórico que englobe también herramientas que no necesariamente tengan un componente virtual, por lo que se hablará de engagement. Finalmente, se proponen una serie de indicadores para su medición y evaluación.

\section{CITIZEN ENGAGEMENT: DEFINICIÓN Y PROPUESTA DE MARCO TEÓRICO}

La aplicación de las TIC ha transformado las zonas urbanas económica, social y espacialmente (Florida, 2003). Sin embargo, el concepto de ciudad inteligente queda lejos de limitarse a la aplicación de las tecnologías dentro de la ciudad, pues se descuidaría el verdadero potencial de la ciudad inteligente: el capital social y sus relaciones dentro del entorno urbano (Albino et al., 2015).

Nuevas formas de relacionarse en el entorno urbano, como es el caso de la gobernanza, afectan a la forma de participar del individuo, y han visto en la ciudad inteligente un escenario idóneo para verse potenciadas a través de distintas iniciativas. Nam y Pardo (2011) aseguran que el apoyo a las políticas de gobernanza es fundamental para implementar estas iniciativas en la ciudad inteligente. Estas políticas muestran cómo los ciudadanos están involucrados con los procesos de toma de decisiones (Bull y Azenoud, 2016). La literatura que analiza este fenómeno habla de engagement.

El engagement es un estado psicológico en el que el individuo disfruta o encuentra satisfacción en la actividad que desempeña, por lo que es más propicio a repetirla. Se trata de un concepto nuclear de la Psicología Positiva que ha sido dotado de base empírica y contrastado en la investigación científica (Salanova y Schaufeli, 2004). Surgió en contraposición al denominado «burnout» o síndrome de «estar quemado» en campos como el laboral o el educativo, y ha sido estudiado y aplicado en distintas áreas, como son el Comercio, la Educación o la Informática, con el objetivo de atraer al sujeto hacia algo y motivarle a que le siga Ilamando su atención (Nahl y Bilal, 2007; O'Brien y Toms, 2008; Webster y Ahuja, 2004). Conviene aclarar que, tal y como señalan Salanova y Schaufeli (2004), la traducción del concepto al español resulta complicada ya que no existe equivalencia terminológica que abarque todos los matices que contiene, por ello, en este trabajo, se utilizará el concepto en su lengua de origen, el inglés.

Optimizando el bienestar psicológico del individuo se obtiene un conjunto de sinergias y beneficios positivos para la comunidad (Bandura,2001; Frierikson, 2002; Salanova y Schaufeli, 2004). Esta idea ha motivado la intención de identificar aquellos factores que pueden provocarle este bienestar al individuo den- 
tro de la actividad participativa. Artículos publicado recientemente sobre esto han comenzado a investigar la aplicación del engagement en el ámbito de la participación ciudadana ya que, en palabras de Castelnovo et al. (2015), «los tradicionales métodos de gobernar no son suficientes para cubrir las demandas de participación ciudadana, debido a las complejidad de un panorama actual lleno de conocimiento tecnológico, confrontación política y conflicto sobre la creación y la gestión de valores públicos».

Sin embargo, esta literatura entiende el engagement como el hecho de involucrar al ciudadano en la toma de decisiones, no existiendo aproximación entre la participación ciudadana y el estado psicológico descritos en líneas anteriores. Pese a que se ha estudiado el comportamiento participativo de los individuos y colectivos en todas sus manifestaciones, y se ha tratado de dar explicación a las motivaciones que llevan a los individuos a participar, hasta la fecha, la literatura sobre engagement en el ámbito de la participación ciudadana no recoge un análisis profundo sobre los aspectos que caracterizan al estado psicológico que puede experimentar un individuo motivado a participar en iniciativas de una ciudad inteligente. En este contexto, la tecnología por sí sola no tiene la capacidad de involucrar al ciudadano, y por ello es importante reparar en aquellos aspectos que, sumados a los tecnológicos, permiten involucrar al ciudadano dentro del espectro de participación ciudadana hasta llevarlo a un estado de engagement (Bryer, 2013).

En este artículo se propone una serie de atributos del engagement que pertenecen de forma intrínseca al sujeto y otros que, por el contrario, pertenecen al sistema que se emplea. Para realizar esta propuesta, en primer lugar, se ha revisado literatura de campos de las Ciencias de la Computación, Psicología y Marketing, y teorías sobre comportamiento humano en el campo de la participación ciudadana. El objetivo ha sido identificar posibles aspectos del engagement que pudieran estar relacionados con los mecanismos de una ciudad inteligente. Los atributos propuestos para el campo de la participación ciudadana en la ciudad inteligente se categorizan en atributos emocionales y atributos sensoriales.

\section{Atributos emocionales}

Emoción es el estado psicológico en el que el sujeto advierte qué valor o qué importancia tiene un asunto determinado dentro de su vida, necesidades o intereses (Yankovic, 2011). Según Yankovic (2011), es la reacción inmediata del ser vivo ante una situación que les puede resultar favorable o desfavorable. En relación a esto se propone identificar los siguientes atributos emocionales:

- Afecto: Stone et al. (2005) definen el afecto como el motivo emocional que lleva al usuario a estar inmerso en un entorno.

- Motivación: Jennings (2000) define la motivación como aquellos elementos que hacen que el individuo se disponga a actuar de alguna forma o a hacer alguna actividad.

Dentro del estudio de estos atributos, la Jerarquía de las Necesidades de Maslow (1954) supuso un cambio de paradigma en la forma de entender qué motiva al sujeto a realizar algo o actuar de alguna forma. Maslow (1954) señala que el individuo tiene un conjunto de necesidades básicas que deben satisfacerse antes que otras. En particular, representa esta jerarquía de forma visual a través de una pirámide, en la que además de un escalón que muestra las «Necesidades Fisiológicas Básicas», se encuentran niveles posteriores que representan las «Necesidades de Seguridad», "Las necesidades Sociales», las «Necesidades de Ego» y en último lugar la "Autorrealización». Así, las necesidades fisiológicas engloban aspectos como el hambre, la sed u otras. Las «Necesidades de Seguridad» son aquellas relacionadas con la seguridad y la protección ante cualquier daño físico y emocional. Las «Necesidades Sociales» incluyen la sensación de pertenencia y aceptación. Las «Necesidades de Ego» son aquellas relacionadas con factores de autoestima como son el respeto hacia uno mismo, los logros o la independencia; y factores provenientes del exterior como el status que consigue uno, el reconocimiento o la atención. Finalmente, las «Necesidades de Autorrealización» encuadran los retos personales, el crecimiento, el potencial de uno y la satisfacción personal.

En este trabajo, tal y como se muestra en la Figura 1, se propone una pirámide basada en el modelo de Maslow (1954) que sirva de base para explicar cuáles son aquellas motivaciones que llevan a un sujeto a participar en los procedimientos de participación ciudadana y que pueden incidir en su nivel de engagement.

En ella se plantean una serie de elementos contenidos en la literatura sobre participación ciudadana (Cross, 2013; Robins, 2007; Rogers, 2013). El objetivo de este modelo es comprender qué motiva a una persona a participar en una iniciativa y así poder medir posteriormente su estado de engagement. Siguiendo la 
lógica de la pirámide, las necesidades que llevan a participar al individuo se representan de forma ordenada en función de la prioridad sobre las necesidades que tiene:

1. Nivel jerárquico de Necesidades Fisiológicas: Un primer nivel representaría las necesidades básicas del ser humano. La motivación para participar del individuo estaría relacionada con las necesidades de asegurarse recursos básicos para la vida como son el acceso a una vivienda, empleo y sustento económico, alimentos, agua, etc.

2. Nivel jerárquico de Necesidades de Seguridad: Son aquellas que entienden tanto la seguridad física como a la psicológica. El individuo participaría motivado por la necesidad de cubrir aspectos como la seguridad ciudadana, un sistema de salud, etc.

\section{FIGURA 1. PIRÁMIDE de JERARQUÍA DE NECESIDADES EN ENGAGEMENT DENTRO DE LA PARTICIPACIÓN CIUDADANA}

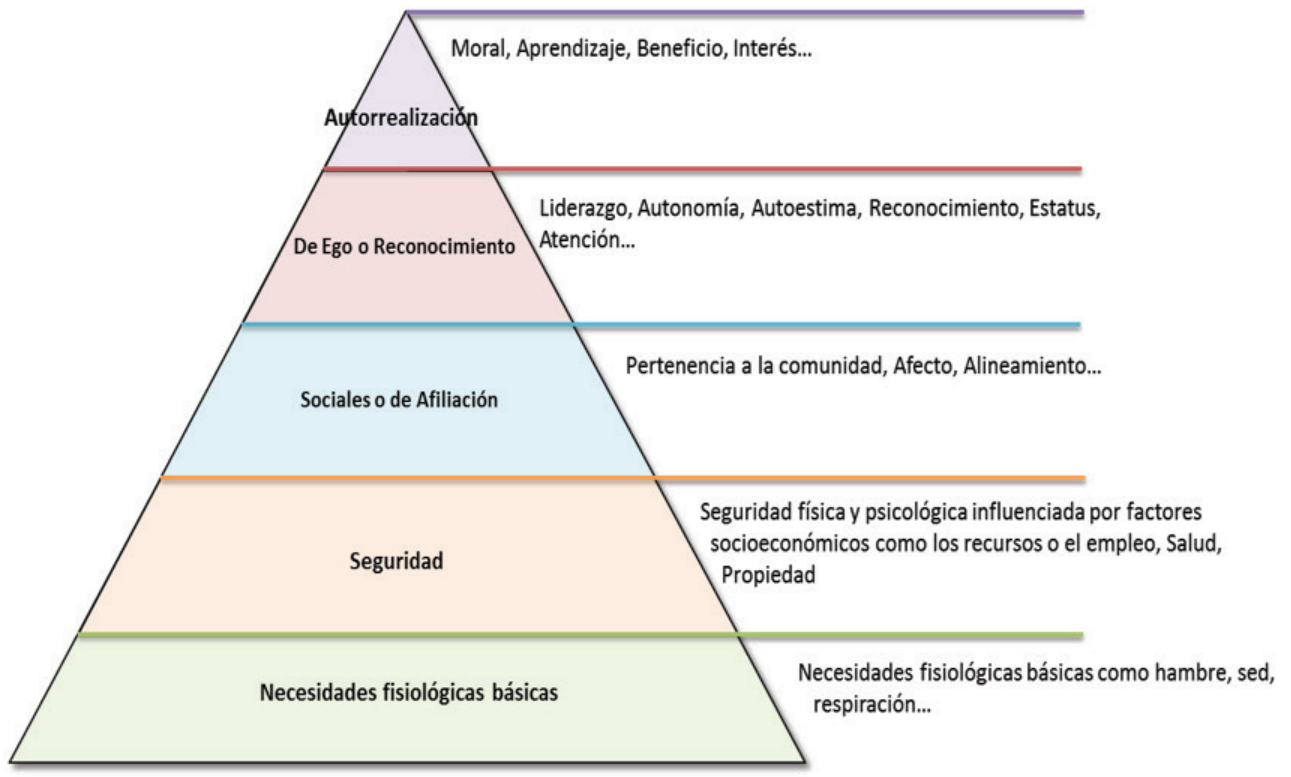

Fuente: Elaboración propia a partir de la jerarquía de necesidades de Maslow (1954).

3. Nivel jerárquico de Necesidades Sociales o de Afiliación: Salanova y Schaufeli (2004) entienden que una de las motivaciones más notables que justifican el engagement en un individuo es el sentimiento que tiene éste de formar parte de un proyecto o de un conjunto de personas. Puede entenderse que en el campo de la participación ciudadana resalte la importancia del individuo de compartir o formar parte de una comunidad, identificando los factores de afiliación y alineamiento político. Se hablaría entonces de identidad colectiva, redes comunes y orden social (Spottke, 2006; Wheelan, 2005).

4. Nivel jerárquico de Necesidades de Ego o Reconocimiento: Otro de los aspectos que motivan al individuo a participar es la percepción de eficiencia política o de que lo que hace «sirve para algo». El individuo de goza de cierto estatus dentro de la comunidad, lo que le dotaría de cierto liderazgo y autonomía dentro del colectivo. Esto puede repercutir en la autoestima del individuo, motivándolo a participar de forma activa en los procesos de participación.

5. Nivel jerárquico de Necesidades de Autorrealización: Se situarían aquí aquellos aspectos que encuadran metas y objetivos personales. En el campo de la participación ciudadana, el individuo puede tener cierta motivación para participar debido a aspectos de corte moral. Se consideran aspectos como la ideología política o las creencias filosóficas o religiosas; es decir, todo aquello que forme parte del ideario del individuo y que despertarían su interés para participar en el procedimiento. Forrester y Watson (1994) hablan también de la competición como mecanismo motivador, entre distintos actores como son los actores políticos, empresariales o civiles, para alcanzar sus intereses. En este contexto, además se ha observado la importancia del aprendizaje del individuo en el campo del engagement, que dependería de las relaciones efectivas entre ciudadanos, empresas y 
gobiernos (Bull y Azennoud 2016; Mezirow, 1994). La colaboración entre actores provee beneficios mutuos de aprendizaje, creando entre ellos una «esfera de influencia» (Bull y Azennoud, 2016). La autorrealización sugiere que el ciudadano que adquiere competencias a través del aprendizaje se siente más comprometido y leal al sistema, teniendo conductas más proactivas y de iniciativa personal. Salanova et al. (2003) indican que individuos con este perfil tienen mayor iniciativa personal así como «niveles más altos de motivación para aprender cosas nuevas y tomar nuevos retos».

Además del afecto y la motivación, se identifican otros atributos emocionales:

- Foco de atención: Matlin (1994) señala el foco de atención como la concentración en la actividad mental. El individuo fija la atención en una actividad que le estimula, y relega a un segundo plano el resto. Salanova y Schaufeli (2004) hablan en este caso de «absorción». Para fomentarlo, el sistema debe procurar tener herramientas orientadas a captar la atención del individuo previamente o durante el transcurso de la actividad participativa.

- Interés: Es el sentimiento que causa una atención especial sobre un objeto o tipos de objeto (O'Brien y Toms, 2008). Las herramientas de participación ciudadana deben tratar de despertar interés en el ciudadano o mantenerlo durante la actividad.

- Vigor: Se caracteriza por altos niveles de energía y resistencia mental. El deseo de invertir esfuerzo lo que se está realizando, incluso cuando aparecen dificultades o imprevistos (Salanova y Schaufeli, 2004).

- Dedicación: Salanova y Schaufeli (2004) señalan que denota alta implicación, junto con la manifestación de un sentimiento de entusiasmo, inspiración, orgullo y reto.

\section{Atributos sensoriales}

Los atributos sensoriales pueden identificarse con la idea de percepción cognoscitiva, mediante la cual los sujetos captan información del entorno. En el engagement estos atributos comprometen al entorno del sujeto a cumplir con una serie de elementos exigidos para que el sujeto encuentre el estímulo que le decida a participar en el procedimiento.

- Aspectos estéticos: Jennings (2000) señala que van referidos a elementos sobre el aspecto de las cosas como la belleza visual, lo natural y lo placentero, pero también la textura o el tacto. En el diseño de las herramientas orientadas a involucrar al individuo en la actividad participativa estos aspectos deben ser cuidados para despertar interés en el sujeto.

- Propiedades atribuidas: estas propiedades están relacionadas con cuestiones sobre apreciación, experiencia y cultura del individuo.

- Feedback: Stone et al. (2005) indican que es la respuesta o reacción del entorno que se comunica al usuario.

- Percepción del tiempo: se trata de la percepción del individuo sobre el tiempo que emplea en realizar una actividad (O'Brien y Toms, 2008).

- Novedad: Aboulafia y Bannon (2004) identifican la novedad como la variedad de retos repentinos o inesperados que sorprenden al individuo, causándole alarma, y que pueden obtenerse de forma visual o auditiva.

- Reto: se trata del esfuerzo invertido en una experiencia (O'Brien y Toms, 2008).

- Control: hace referencia al sentimiento que se desarrolla sobre la experiencia (O'Brien y Toms, 2008).

- Eficacia: según Bandura (1997), la eficacia y autoeficacia son las creencias en las propias capacidades para organizar y ejecutar los cursos de acción requeridos para producir logros. Atendiendo a la Teoría Social Cognitiva, estas creencias inciden en la forma de actuar, pensar y sentir del individuo, proporcionando a la persona un mecanismo auto-motivador. Según Salanova y Schaufeli (2004), al observar las propias competencias, la persona se impone a sí misma metas, con lo que ello comporta la movilización del esfuerzo, dirección y persistencia en el tiempo. Se relaciona también con costes como la cantidad de tiempo que se invertirá en realizar algo; Bajos niveles de eficacia llevan asociados abandonos de la actividad que se está realizando.

Parte de estos atributos son características que no son intrínsecas al individuo, sino que pertenecen al sistema y a la relación de éste con el individuo. Por ello, estas características deben ser diseñadas en las 
distintas herramientas de participación para facilitar al individuo un estado de engagement. Estas características son: aspectos estéticos, foco de atención, interés, novedad, feedback, reto y control. Como ejemplo, un sistema que no suponga reto alguno para el individuo como un sistema que muestre un grado de dificultar elevado, y por lo tanto un reto inalcanzable, no fomentarán el engagement de la persona. El hecho de cuidar cada uno de estos aspectos de manera óptima presenta mayor probabilidad de que aumentar la participación y atracción del individuo.

\section{FASES DEL ENGAGMENT}

El engagement es un estado psicológico no puntual. Es un proceso por el que el individuo discurre en varias fases con distinta intensidad. Se propone un modelo de fases del engagement para el ámbito de la participación ciudadana.

\subsection{Punto de partida o introducción del engagement}

El engagement puede comenzar por distintos factores que motiven al ciudadano. El ciudadano puede estar interesado en un aspecto particular, como por ejemplo cierta política pública. Este interés correspondería a una motivación del sujeto dentro de las Necesidades de Autorrealización. Además, puede entrar en juego la existencia de la Necesidad de Socialización del individuo: pueden existir motivaciones sociales para interactuar con otras personas.

El entorno y las recomendaciones de otros individuos pueden despertar en el sujeto interés por participar en algún procedimiento en concreto. La información juega un papel fundamental, por ello deben cuidarse aspectos tanto estéticos, como los referentes al discurso o relato político. Los poderes públicos deben hacer un mayor esfuerzo para despertar el interés del ciudadano y hacerlo partícipe del procedimiento de toma de decisiones. La realización de una segmentación de mercado identificaría los segmentos de población que componen el mercado y podrían seleccionarse aquellos a los que nos interesa dirigirnos prioritariamente. Además, debe gestionarse la diversidad. Las administraciones deben gestionar estas estrategias de mercado para dirigirse a un público objetivo en función de las diferencias culturales, los estilos de vida, actitudes, creencias, valores, comportamientos y motivaciones (Salanova y Schaufeli, 2004).

\subsection{Mantenimiento de engagement}

Uno de los atributos de engagement fundamentales en esta fase es la novedad. Al ciudadano le impacta una nueva experiencia de participación que debe ser estimulada a través de la incorporación de elementos nuevos, interesantes e inusuales.

El ciudadano ha centrado su foco de atención en la actividad participativa y experimenta altos niveles de atención y concentración (Matlin, 1994). El nivel de interés incrementa y comienza a percibir una serie de retos. A estos retos se suma una sensación de control y éxito o satisfacción al comprobar que el propio individuo cuenta con herramientas y la capacidad de hacer frente a esos retos. Debe atenderse, por tanto, a la complejidad del procedimiento participativo y a la accesibilidad al mismo. Es importante que el procedimiento participativo no resulte tedioso y largo, cuidándose estos aspectos.

La comunicación es relevante, pues uno de los aspectos con mayor peso que influyen en esta fase de engagement es el «feedback» o respuesta, en este caso, de los poderes públicos que tienen hacia el ciudadano. Este feedback se trata fundamentalmente de la información que se le comunica al ciudadano sobre elementos como los pasos del procedimiento, las acciones a realizar, los resultados que se obtienen y los fines que se persiguen con cada procedimiento. Acciones para promocionar y dar visibilidad al procedimiento de participación son recomendables para que el individuo mantenga su interés en el proceso.

\subsection{Disengagement}

Puede experimentarse un descenso del nivel de interés o incluso cese del engagement. Esta situación se da cuando el ciudadano detiene la realización de una actividad o cuando existen factores externos en el entorno del ciudadano que interrumpen el engagement (O'Brien y Toms, 2008). Puede alcanzarse una fase de saturación en el que el interés del individuo declive, bien porque se conozca el procedimiento y se haya 
tomado la decisión de no participar más, bien porque se haya alcanzado el techo de aceptación del mismo. Es importante diseñar estrategias para revitalizar ese estado psicológico y volver a iniciar un nuevo ciclo de engagement con el fin de evitar la cristalización del proceso participativo y su desaparición a causa de falta de ciudadanos que participen o para evitar que nazca en el individuo la indiferencia.

\subsection{Reengagement}

Esta fase se experimenta cuando no se ha puesto fin de forma definitiva al engagement. Puede suceder que cese temporalmente el proceso participativo, como sucede en casos como los procedimientos de presupuestos participativos, y que se reanude transcurrido un tiempo. En este caso, el engagement puede cesar cuando cese esa actividad participativa, y volver en un periodo de tiempo (O'Brien y Toms, 2008). El individuo volvería a experimentar engagement cuando esté predispuesto psicológicamente para ello, bien porque cese la distracción externa que propicie la pérdida de atención en los procedimientos de participación ciudadana, bien porque recupere las herramientas que le permitan participar.

Aquí es importante el atributo experiencia. El recuerdo del ciudadano de que la participación haya resultado una experiencia placentera, satisfactoria, con la que se haya disfrutado, influirá en el hecho de que vuelva a participar. Si por el contrario, la participación supuso para el ciudadano sensaciones de incertidumbre, duda, inseguridad o frustración, no se planteará volver a participar en el procedimiento. El exceso de información o la falta de feedback por parte de los poderes públicos contribuyen al rechazo del ciudadano hacia el procedimiento.

\subsection{No engagement}

Existe la posibilidad de que el ciudadano no llegue a experimentar engagement (O'Brien y Toms, 2008). Puede que no lo experimente ya que psicológicamente no esté preparado. Así, la participación ciudadana no entraría dentro de las experiencias del individuo, bien por indiferencia, por falta de tiempo, o bien por los costes de participar como por ejemplo la distancia.

La ausencia de engagement puede proceder del propio sistema de participación generando experiencias no disfrutables o divertidas, siendo un sistema difícil de usar, con contenido poco atractivo y con exceso de información. El diseño o la estrategia del procedimiento y de sus canales de participación son clave para que el individuo no se muestre indiferente ante la participación. Estudios muestran como un uso adecuado de los medios sociales, por ejemplo, incrementan en diez el número de participantes en los debates públicos (Castelnovo et al., 2015).

\section{PARTICIPACIÓN CIUDADANA EN LAS CIUDADES INTELIGENTES: REVISIÓN DE HERRAMIENTAS DE PARTICIPACIÓN}

Brugué et al. (2003) señalan que una ciudadanía culturalmente más preparada y sofisticada ha aparecido en las sociedades (Inglehart, 1991). Esta ciudadanía experimenta ansias por encontrar vías alternativas de expresión participativa (Budge, 1996). La desafección y un descontento de los ciudadanos hacia las instituciones es otro de los acontecimientos determinantes para un cambio social (Kaase y Newton, 1995). La ciudadanía ha dejado de confiar en las tradicionales formas de implicación política y demanda nuevas formas a través de las cuales puedan tener una relación más directa con la gestión de los asuntos públicos (Martins, 2002). Además, la irrupción de las TIC han jugado un papel fundamental dentro de los cambios socioculturales por los que atraviesa una sociedad cada vez más compleja (Subirats, 2013). Una sociedad en red en la que las relaciones sociales están basadas en las redes tecnológicas (Castells 2000).

Las sociedades han ido demandando no sólo una mayor participación, sino que se han redistribuido el poder entre todos los actores participantes. Las formas de participación ciudadana se ven ampliadas, complementadas o en su caso sustituidas, por nuevas reglas de juego. En las últimas décadas, la concepción de prestación de servicios y toma de decisiones reservada a las élites se ha reinterpretado. La política es entendida ahora como producto de las interacciones entre numerosos actores, y no está reservada únicamente a los gestores públicos en procesos «top down» (Bovaird, 2007). Se asiste por tanto a una reorganización del control público y redistribución de poder (Meijer, 2016). Surgen nuevas formas de entender la gestión de lo público en las que la ciudadanía adquiere un nuevo rol. Tal y como señalan Andrés y Bonivento (2009), 
los gobiernos pasan de ser el único actor que ejerce competencias públicas para ser un coordinador entre numerosos actores.

Meijer y Bolívar (2015) señalan que una ciudad inteligente significa elaborar nuevas formas de colaboración humana a través de las TIC. Las idea de que las ciudades inteligentes están dotadas con infraestructuras urbanas de alta tecnología, conductoras de la información y que analizan big data gana fuerza dentro de la literatura (Niederer y Priester, 2016). Son ciudades equipadas con herramientas y capacidad de organización, en las que los ciudadanos participan en lo que se ha denominado «city making» (Brynskov et al., 2014). Estas herramientas permiten que los ciudadanos sean parte de los procedimientos de toma de decisiones dentro de la ciudad inteligente. Las TIC facilitarían así la transición de una forma de gobierno tradicional a distintas formas relacionales de gobierno como son la gobernanza, la e-participación y la coproducción, cambiando los roles de los poderes públicos con los ciudadanos debido a la transformación y al impacto que ha tenido la conectividad ubicua de la informática urbana (Colombo, 2006; Anttiroiko, 2016). Los autores advierten que la tecnología por sí sola no hace a una ciudad que sea inteligente. Se requiere de políticas que entiendan de tecnología, a la vez que de un plan estratégico que ponga el foco en otros valores y recursos públicos como los económicos, humanos y materiales (Meijer y Bolívar, 2015; Nam y Pardo, 2011; Ramilo Araujo y Fernández Rupérez, 2012). Además, tal y como señalan Ramilo Araujo y Fernández Rupérez (2012), debe tenerse en cuenta una serie de implicaciones existentes a la hora de diseñar un proceso participativo como las barreras existentes a la hora de seleccionar canales de participación y los esfuerzos de comunicación que deben realizarse para poder difundir a la ciudadanía la iniciativa de participación.

Es necesaria la identificación de las herramientas que pueden encontrarse en la ciudad inteligente para ser utilizadas como canales de participación ciudadana. Para ello, debe comenzarse describiendo aquellas tecnologías que alberga la ciudad inteligente y sobre las que se basan las herramientas de participación ciudadana.

Existen tecnologías que componen las infraestructuras y dotan a una ciudad inteligente de funcionalidad a varios niveles (Salim y Haque, 2015). La ciudad inteligente reparte su tecnología en distintas capas con el objetivo de producir, recoger, transmitir y visualizar datos. Así, dentro de un primer nivel se encuentran sensores físicos que se distribuyen por la ciudad y recogen datos de diferente índole, como medidas relativas a localizaciones (mediante señales GPS), contaminación, ruido, temperatura y humedad, entre otros. Estos datos recogidos pasan a un segundo nivel compuesto de dispositivos electrónicos, como smartphones, relojes inteligentes y sistemas integrados en vehículos. En general, estos dispositivos transmiten los datos recogidos a través de Internet mediante conexiones WiFi, Bluetooth, o vía satélite. El conjunto de estos dispositivos es lo que se conoce como el «Internet de las Cosas» o Internet of Things (IoT). Internet corresponde a un tercer nivel en el que esos datos están disponibles para ser utilizados por distintas herramientas o aplicaciones. Algunos ejemplos son aquellas sobre el control inteligente de tráfico, consumo energético eficiente, control de nivel de contaminación del aire, calidad del agua, gestión inteligente de basuras, detección de incendios y parking inteligente. En este trabajo nos centramos en las destinadas a la participación ciudadana que pueden encontrarse en una ciudad inteligente, muchas de las cuales utilizan las tecnologías descritas. Estas herramientas se han clasificado en dos tipos en este trabajo: herramientas físicas y herramientas virtuales.

\section{Herramientas físicas}

Esta clasificación engloba todas aquellas herramientas que responden a un componente físico que permite la participación. Salim y Haque (2015) identifican las siguientes:

- Sondas urbanas: Paulos y Jenkins (2005) definen las sondas urbanas como un conjunto de artefactos situados en las calles que incitan a los ciudadanos a participar en el diseño de la ciudad. Al estar al alcance del ciudadano, le permiten interactuar con ellas con el fin de informarle o consultarle acerca de las decisiones que deben tomarse en relación a distintos proyectos de la ciudad (Dalsgaard y Eriksson, 2013).

- Monitores públicos (displays), pantallas urbanas y fachadas interactivas: estos elementos de mobiliario urbano permiten al ciudadano visualizar información por toda la ciudad. La diferencia fundamental entre los tres elementos se debe al tamaño de las pantallas, la localización de las mismas y el tipo de contenido proyectado en cada dispositivo (Salim y Haque, 2015).

- Pantallas públicas interactivas: son usadas para comunicar información al ciudadano de forma directa en los espacios públicos (Salim y Haque, 2015). Además, a diferencia de los monitores públi- 
cos, explicados anteriormente, estas pantallas pueden ser utilizadas para obtener respuesta de la ciudadanía sobre algo (Schroeter, 2012).

- Aplicaciones basadas en Internet of Things: el hecho de hacer accesible Internet de las Cosas a los ciudadanos permite una participación ciudadana a gran escala (Paulos et al., 2008) Iniciativas públicas abiertas para compartir datos a tiempo real hacen que los ciudadanos, aun no siendo expertos, puedan llegar a participar en iniciativas de crowdsourcing ${ }^{1}$ y crowdsensing ${ }^{2}$ (Salim y Haque, 2015).

- Plataformas: Anttiroiko (2016) señala la existencia dentro de la ciudad inteligente de plataformas no sólo virtuales, sino que pueden ser físicas, tecnológicas o sociales. Estas plataformas permiten la construcción de procesos socio-técnicos o económicos gracias a la interconexión e interacción de los distintos actores (Anttiroiko, 2016). Anttiroiko (2016) indica que las plataformas permiten el acceso a los individuos y los involucra, facilitan el diálogo, la convergencia de ideas, la toma de decisiones y la integración política.

- Living labs: conciben la ciudad como una plataforma a gran escala (Anttiroiko, 2016). Pueden ser diseñados como regiones físicas o realidades virtuales donde los usuarios co-crean de forma innovadora. Desarrollan productos y proyectos gracias a las herramientas y métodos que se ponen a disposición en la ciudad. La Comisión Europea (The Helsinki Manifesto, 2006) los caracteriza como Alianzas Público-Privado-Personas cuyos actores involucrados tienen cuatro objetivos principales: co-crear, explorar, experimentar y evaluar los productos o proyectos experimentados.

\section{Herramientas virtuales}

Las herramientas virtuales son aquellas que utilizan tecnologías como Internet como elemento principal. Pueden identificarse las siguientes herramientas (Ramilo Araujo y Fernández-Rupérez, 2012; Mandarano et al., 2010):

- Portales Web de las administraciones públicas: Son las herramientas de comunicación al ciudadano por excelencia. La información que se publica va dirigida a un público genérico e indefinido. Para potenciar su utilidad, suele ir combinada con otras herramientas.

- Q\&A (Questions \& Answers): los ciudadanos pueden enviar sus dudas y preguntas a los administradores de un portal Web. Se trata de una herramienta indicada para que los ciudadanos expongan sus dudas, recibiendo feedback directo de las administraciones.

- Mashups: Son un tipo de portales Web que van combinados con otras tecnologías como son por ejemplo aplicaciones que combinan mapas interactivos con datos estadísticos.

- E-Mail: Supone una herramienta de comunicación de bajo coste para los usuarios.

- Mensajes de texto móviles (SMS) y mensajería instantánea (p. ej., WhatsApp): se utilizan con frecuencia debido a que permiten la comunicación de persona a persona o interactuar con sistemas automáticos.

- RSS (Really Simple Sindication): Permite al usuario recibir información en su e-mail sobre el contenido de algún portal Web. Los mensajes se reciben cuando el contenido de la página web se actualiza. Es una herramienta útil para que el ciudadano se mantenga siempre informado.

- Social media: Social media o medios sociales son todos aquellos sitios Web en los cuales los usuarios pueden compartir información o contenido multimedia. Es contenido social porque los ciudadanos pueden compartir información y comentar e interactuar con otros ciudadanos (Butteriss et al., 2012).

- Wikis: Las páginas Wiki son aquellas en las que los individuos crean contenido objetivo sobre hechos, personas o lugares, y que pueden editarse por cualquier persona (Mandarano et al., 2010).

- Blogs: Es un tipo de Web administrada y mantenida por un conjunto de personas que generan o intervienen a través de artículos o «posts» sobre temas concretos. Pueden a su vez combinarse con otras herramientas como el contenido multimedia (Mandarano et al., 2010).

- Multimedia sharing: Los ciudadanos pueden realizar contenido multimedia como video, imágenes o audios a través de dispositivos como sus smartphones personales. Este conte-

\footnotetext{
1 El crowdsourcing es el acto de externalización de herramientas o recursos para desarrollar algo nuevo a través de una llamada abierta a los ciudadanos (Howe, 2006).

2 Las plataformas de crowdsensing permiten utilizar la información obtenida, a través de los datos recogidos por sensores integrados en distintos dispositivos de computación ubicua como smartphones (SALIM y HAQUE, 2015).
} 
nido multimedia es compartido en la Red y permiten al individuo obtener feedback de otros individuos.

- Microblogging: Siguen la dinámica de los Blogs pero con un espacio escritura limitada. El ejemplo más claro es Twitter, que es utilizada como herramienta útil para distribuir información e interactuar con un límite de 280 caracteres por intervención.

- Formularios Web: este tipo de formularios facilitan la tarea encuestar, sustituyendo los cuestionarios en papel o de forma telefónica. Son una herramienta útil para consultar al ciudadano sobre temas concretos. Pueden distribuirse a través de distintas formas como son el e-mail y los portales Web.

- Online Social Networking sites: El elemento esencial es el ciudadano, el cual interactúa con sus contactos (Butteriss et al., 2012). Se diferencian de los medios sociales en que en estos últimos el elemento esencial es el contenido que se genera y se comparte por el usuario. En el ámbito de las políticas públicas, estas herramientas son útiles para informar, educar e involucrar a los miembros de la comunidad (Mandarano et al., 2010). Ejemplos de estos sistemas son:

- Redes sociales online: Permiten la interacción del ciudadano con sus contactos. Ejemplos de ellas son Facebook y Linkedln. El empleo de las redes sociales online en las relaciones gobiernociudadanos (G2C) es un área de investigación que ha despertado el interés de distintos autores (Bonsón et al., 2012, 2015; Mergel, 2013; Norris y Reddik, 2013).

- Encuentros virtuales o foros: Pertenecientes a la categoría de grupos de discusión online, permiten reducir el coste que supone a los usuarios acudir a un sitio de forma presencial para participar en algo. Encuentros virtuales como los foros permiten la concurrencia de numerosos individuos que debaten o comparten información de forma virtual. Los ciudadanos pueden participar sin coste y sin límite en la intervención.

- Plataformas virtuales: Son aquellos soportes virtuales que permiten la concurrencia de distintos individuos y la participación a gran escala y con costes reducidos (Anttiroiko, 2016). Algunas iniciativas que pueden desarrollarse a través de plataformas virtuales son las siguientes:

- Plataformas de participación habilitadas por las administraciones públicas: son aquellas plataformas virtuales que las administraciones públicas ponen a disposición de los ciudadanos con el objetivo de que éstos participen en una iniciativa. Un ejemplo de esto son las plataformas de participación ciudadana que distintos ayuntamientos habilitan para los procedimientos de presupuestos participativos.

- Plataformas de crowdsourcing: A través de estas plataformas los ciudadanos reúnen recursos destinados a la realización de alguna actividad o servicio (Howe, 2006). Pueden utilizar dispositivos como smarthphones no solo para compartir información, conocimiento u opinión sino que puede utilizar sensores de información como los de localización, con el fin de acercar al ciudadano a las iniciativas de su entorno más cercano (Salim y Haque, 2015).

- Plataformas de crowdsensing: La información obtenida a través de los datos recogidos por sensores integrados en distintos dispositivos de computación ubicua es utilizada por distintos actores críticos para hacer estudios de diversa índole como por ejemplo estudios de mercado en una localización concreta.

- PPGIS o Sistemas de Información Geográfica de Participación Ciudadana: permiten generar y gestionar información sobre localización. La información es utilizada en el campo de la planificación por los individuos y comunidades. Esta información puede ser sobre edificios construidos, basuras, espacios vacíos, etc. Además de obtener el mapa de información de los individuos sobre factores sociales, económicos y demográficos también son idóneas para obtener distinta información que contribuye a que los distintos actores participen más preparados en los procedimientos de toma de decisiones (Sieber, 2006).

- Herramientas de Gamificación ${ }^{3}$ : en las ciudades inteligentes pueden darse, tanto de forma física o presencial como de forma virtual, distintos mecanismos de Gamificación. Opromolla et al. (2015) proponen el uso de estos elementos de gamificación en el ámbito de la participación ciudadana con

3 La gamificación consiste en usar elementos de juego en otros contextos (OPROMOLLA et al., 2015). 
el objetivo de involucrar al ciudadano de distintas maneras. Para ellos, el objetivo de la gamificación aplicada al contexto de la ciudad es concienciar e involucrar al ciudadano en los asuntos de su entorno. Identifican distintos tipos de jugador y roles posibles, objetivos de juego y distintos elementos como son las reglas del juego, y el «masten» del juego o director.

\section{PROPUESTA DE HERRAMIENTAS PARA CADA NIVEL DE PARTICIPACIÓN CIUDADANA}

Tan y como señalan Ramilo Araujo y Fernández Rupérez (2012): «Hay que tener en cuenta qué tipo de participación permite cada herramienta». Para poder abordar esta cuestión, se ha escogido el modelo de la OCDE $(2001 ; 2003)$ sobre los distintos niveles de interacción y participación existentes entre la administración y ciudadanía, con el fin de identificar y encuadrar en cada nivel del modelo de OCDE $(2001 ; 2003)$ las herramientas más recomendadas para la participación de acuerdo a las características que las definen.

\section{Nivel de Información}

Según la OCDE $(2001 ; 2003)$, el nivel de información se entiende como una relación unidireccional que va desde las instituciones públicas hacia los ciudadanos. La OCDE $(2001 ; 2003)$ define esta relación entre las instituciones y los ciudadanos como la dinámica en la que «Las instituciones públicas difunden, por iniciativa propia, informaciones sobre elaboración de las políticas, o bien los ciudadanos obtienen información a partir de su propia demanda».

La OCDE (2001) habla de herramientas como los Diarios Oficiales. En una ciudad inteligente, las herramientas que pueden ser utilizadas en este nivel de participación ciudadana por parte de la Administración debido, entre otros aspectos, a las similitudes con la descripción y ejemplos que ofrece la OCDE (2001; 2003) son las siguientes:

- Sondas Urbanas, Monitores, Pantallas Urbanas Interactivas.

- E-Mail, SMS y Mensajería Instantánea.

- Portales Web, Mashups, Q\&A, RSS.

- Wikis y Blogs, Microblogging y Online Social Networking sites, Multimedia Sharing sites, PPGIS.

Las herramientas físicas que pueden utilizarse en este nivel de información son las sondas urbanas, los monitores y las pantallas públicas interactivas. Estas tienen como función captar la atención o el interés del ciudadano a través de los aspectos estéticos, por lo que en estas herramientas deben cuidarse en mayor medida. Tanto los aspectos estéticos como la novedad de la experiencia predicen el foco de atención (O'Brien y Toms, 2008). Por otro lado, tanto las sondas urbanas, como las pantallas públicas interactivas ofrecen al usuario cierto feedback, que contribuiría a involucrar al ciudadano hasta el nivel de engagement (Kukka et al., 2014).

En cuanto a las herramientas virtuales, en este nivel las administraciones pueden informar al ciudadano a través de los Portales Web, Mashups, PPGIS, Q\&A, E-Mail, SMS, RSS, Multimedia Sharing, Blogs y Wikis, Microblogging y Online Social Networking sites. Debido a sus características, Portales Web, Mashups, PPGIS, Q\&A, E-Mail, SMS y RSS.

Herramientas de Multimedia Sharing, Blogs y Wikis, Social Networks y Foros tendrían el potencial de estimular atributos como el de identidad colectiva al facilitar que el sujeto forme parte de un colectivo o un grupo virtual, relacionándose con otros sujetos o por formar parte de un público objetivo al que la Administración se dirige.

En relación con los aspectos estéticos, Portales Web, Mashups, PPGIS, E-Mail, Multimedia Sharing y Social Networks, los cuidarían en mayor medida frente a otras herramientas debido a sus características.

En el nivel de información, el feedback entre la Administración y ciudadanos puede ser facilitado en mayor medida por las herramientas virtuales de Q\&A, E-Mail, SMS, RSS, Multimedia Sharing, Blogs y Wikis, Microblogging y Online Social Networking sites. Se debe a que estas herramientas permiten al usuario demandar información a la Administración de manera más sencilla para satisfacer su derecho a la Información.

Atributos como el control, el reto y la competición no tendrían relevancia pues en este nivel de participación el ciudadano permanece más pasivo a diferencia de otros niveles. 


\section{Nivel de consulta}

En este nivel de participación, la OCDE $(2001 ; 2003)$ define la relación entre administración y ciudadano como una relación bidireccional limitada. Las instituciones públicas demandan y reciben respuestas de los ciudadanos sobre la elaboración de políticas públicas. Para ello, son las instituciones públicas las que seleccionan de qué interlocutores desean recibir una opinión y puntos precisos sobre los que van a opinar. Pese a que la OCDE $(2001 ; 2003)$ limita este nivel a la mera consulta, este trabajo propone enfocar este nivel de participación a la co-participación ciudadana, en la cual la administración orquesta el proceso participativo y además, tiene la última palabra tanto en la definición de una política como en la decisión final.

La OCDE (2001) señala como ejemplos de herramientas, en este nivel de participación basado en la consulta, las encuestas de opinión pública o los procesos de diálogo. Las herramientas que proponemos que se pueden encontrar en una ciudad inteligente para este nivel de participación son las siguientes:

- Sondas Urbanas, Monitores, Pantallas Urbanas Interactivas, Plataformas, Living labs.

- E-Mail, SMS y Mensajería Instantánea.

- Wikis y Blogs, Microblogging and Online Social Networking sites, Multimedia Sharing sites, PPGIS.

- Plataformas de Participación Online, Foros, Formularios web.

- Herramientas de «Gamificación».

Las Sondas Urbanas, Pantallas Públicas Interactivas, Multimedia Sharing sites, Wikis y Blogs, Online Social Networks, Plataformas de Participación Online y Mecanismos de Gamificación pueden diseñarse para que sean provocativas e interesantes para el ciudadano (Carr et al., 1992; Memarovic, 2012).

La consulta implica una posición más activa en el ciudadano en comparación con el nivel de información por lo que atributos como el reconocimiento, la autoestima, el estatus, el liderazgo el control, el reto o la competición son relevantes para el individuo. Además, el ciudadano puede percibir en mayor medida formar parte de algún proyecto y comunidad. Es decir, puede surgir cierta identidad colectiva.

Whyte (1980) indica que, añadiendo un reto a la actividad, el individuo llegaría a experimentar engagement. En tal caso, las herramientas que facilitarían en mayor medida la regulación de cierta dificultad en la actividad son las Plataformas de Participación, los Living labs, los Formularios web, las Plataformas de Participación Online y los Mecanismos de Gamificación.

De igual forma sucede con el feedback (Kukka et al., 2014). Las herramientas que potenciarían ese diálogo entre la Administración y el ciudadano en mayor medida son las Pantallas Públicas interactivas, las Plataformas de Participación, los Living labs, el Microblogging, las Online Social Networks y los Foros.

Algunos autores han señalado algunos aspectos sobre el impacto de las redes sociales en el ciudadano. En ellas, los ciudadanos participan en mayor medida cuando se trata de temas a nivel local o que afectan directamente a su entorno (Bonsón et al., 2015; Lev-On y Steinfeld, 2015).Esto nos lleva a plantearnos la posible relación que pueda existir con algunos atributos del engagement como las necesidades de seguridad.

\section{Nivel de delegación de poder}

La OCDE $(2001 ; 2003)$ habla del Nivel de participación activa. Sin embargo, en este trabajo se le ha asignado otra denominación a este nivel, pues a nuestro entender todos los niveles, de una forma $u$ otra, implican participación ciudadana, por lo que lo denominaremos en este trabajo como nivel de delegación de poder.

La OCDE $(2001 ; 2003)$ indica que se trata de una relación bidireccional entre las instituciones y los ciudadanos. Los ciudadanos participan activamente en la toma de decisiones y en la elaboración de políticas públicas. Estos juegan un rol en la elaboración de políticas públicas; por ejemplo, proponiendo alternativas. Aunque la OCDE $(2001 ; 2003)$ indica que la decisión final sigue siendo del gobierno, este trabajo propone enfocar este tercer nivel a los mecanismos de co-producción y gobernanza. Aversano et al. (2013) señalan que cada vez más ciudadanos participan en los sistemas de gobernanza de la ciudad y juegan un papel más principal en la gestión de lo público a través de fórmulas como la coproducción y la coevaluación de servicios, por lo que es necesario reestructurar el esquema participativo para incluir estas nuevas formas de participación. Las herramientas recomendables para este nivel de participación son las siguientes:

- Living labs.

- Wikis y Blogs, Microblogging y Online Social Networking sites, Multimedia Sharing sites. 
- Plataformas de Participación Online, Foros, Plataformas de Crodwsourcing, Plataformas de Crowdsensing (loT).

- Mecanismos de gamificación o de juego.

Las plataformas permiten la interacción de los individuos para poder contribuir a idear, entender a los usuarios y a sus contextos, experimentar con prototipos, etc. (Anttiroiko, 2016). Así, tanto las Plataformas de Participación y Living labs como las Plataformas de Participación Online y las de Crowdsourcing pueden incidir en el engagement, permitiendo la gestión de figuras como la co-producción y el co-diseño. Además, los Mecanismos de Gamificación ofrecen una serie de características y dinámicas que pueden estimular a la mayor parte de los atributos del engagement.

El hecho de que la iniciativa resida en los ciudadanos, el hecho de formar parte de un grupo o un proyecto, de adquirir un rol frente a otros actores involucrados o una función; suponen la interacción de ciertos atributos como son el reconocimiento individual, el estatus, el liderazgo, el aprendizaje, eficacia, autonomía, identidad colectiva, reto, control y competición. Esto puede deberse en parte, a que se da un escenario en el que el ciudadano adquiere más responsabilidad y un mayor control sobre las herramientas.

\section{IMPACTO DE LAS HERRAMIENTAS DE PARTICIPACIÓN SOBRE LOS ATRIBUTOS DEL ENGAGEMENT}

En relación con el posible impacto de las herramientas de participación ciudadana de la ciudad inteligente sobre los atributos del engagement se han identificado una serie de cuestiones comentadas a continuación.

En primer lugar, en cuanto a los atributos intrínsecos al sujeto, atributos como el reconocimiento individual se potenciarían con medidas como la personalización de mensaje, dentro del nivel de información. Esto motivaría la percepción de que la Administración se dedica al ciudadano directamente a través de una serie de canales más personales, como serían los E-Mail y los SMS. En los niveles de consulta y participación, por el contrario, se entendería el reconocimiento como la identificación del sujeto con su rol como actor crítico, por lo que las herramientas en las que se puede hacer más patente esto son aquellas como las Plataformas o los Living labs. La "gamificación» supone también una dedicación personal al ciudadano ya que busca atraerlo y empoderarlo mediante lógicas de juego, y eso conllevaría a diseñar estrategias más específicas para distintos segmentos y perfiles de «jugadores».

La autoeficacia reside en la creencia del individuo de estar capacitado para organizar los cursos de la acción a la hora de producir o alcanzar determinados logros. Aquellas herramientas que permiten al usuario generar su propio contenido en distintas plataformas, bien tomar la iniciativa bien aportar su punto de vista o experiencia serían las indicadas. La autonomía o independencia encontrarían dificultad de fomentarse en el nivel de consulta, pues el ciudadano no toma una iniciativa plena ya que el objetivo es ser consultado por la Administración. Esta independencia, en cambio, sí se produciría en el nivel de delegación de poder en el ciudadano y por casi todas las herramientas de participación.

La autoestima encuentra cierta dificultad para ser relacionada con distintas herramientas. Se ha entendido como una consecuencia derivada de otros atributos por lo que, al igual que en el resto de relaciones, son necesarios análisis posteriores a la participación del ciudadano para comprobar si se dan relaciones de causalidad entre herramienta y atributo.

Sentirse parte de algo es un atributo notable en el engagement. El individuo se siente parte de un colectivo o de un proyecto conjunto. Múltiples herramientas pueden contribuir a que el individuo tenga esa percepción. Se destaca el potencial de las herramientas virtuales para perseguir este fin como son las Online Social Networks y los Foros.

El estatus y el liderazgo sólo pueden ser motivados, por definición, en los niveles de consulta y delegación de poder en el ciudadano. Dentro de éstos, casi todas las herramientas en las que el ciudadano tiene un papel activo y en las que genera contenido, pueden verse afectados estos atributos. Las métricas empleadas para medir estos atributos pueden ser por ejemplo los «like» o «me gusta» que ofrecen algunas herramientas con el fin de que el resto de usuarios valoren los contenidos. Además, en las herramientas físicas, estos atributos pueden verse potenciados con algunas como las Plataformas Físicas o los Living labs, en los que la interacción dentro de grupos o sectores pueden dotar al ciudadano de cierto liderazgo en el desempeño de la actividad y de estatus dentro del colectivo o grupo de actores con los que se relaciona. 
En cuanto a los atributos pertenecientes al sistema y que afectan a la psicología del individuo, comentar que las herramientas virtuales pueden cuidar elementos como los aspectos estéticos, el interés, el control y el reto (O'Brien y Toms, 2008). En el nivel de información, el control y el reto no pueden ser aplicados ya que por definición el ciudadano no se enfrenta a ellos. En el nivel de consulta, en cambio, el control y el reto son afectados en mayor medida a las pantallas urbanas de interacción, a las plataformas de participación físicas y living labs, a los Formularios web, las Plataformas de Participación Online y a los Mecanismos de Gamificación. En el nivel de delegación de poder, el reto y el control son cubiertos por casi todas las herramientas señaladas para este nivel.

El feedback se daría, dentro del nivel de consulta en las Pantallas Públicas Interactivas, las Plataformas de Participación físicas y los Living labs, Microblogging y Online Social Networking sites, los Foros y algunos Mecanismos de Gamificación. En el nivel de información sólo en aquellas herramientas preparadas para la interacción de la Administración y del ciudadano a través de las demandas de información de este como son el Q\&A, los E-Mail y SMS, Microblogging y Online Social Networking sites.

\section{MEDICIÓN DE ATRIBUTOS DE ENGAGEMENT EN HERRAMIENTAS DE PARTICIPACIÓN}

En este trabajo se han propuesto cuestiones que afectan al engagement del ciudadano y a las herramientas que éste utiliza para participar en cada uno de los niveles establecidos. Resulta necesario validar estas teorías en trabajos futuros mediante la realización de un estudio explicativo que muestren la existencia o no de la correlación entre los distintos atributos del engagement, y la existencia o no de causalidad entre las distintas herramientas y dichos atributos. Se propone un marco de evaluación para estos objetivos.

Interesa medir el número de individuos que llegan al estado de engagement $\mathrm{y}$, además, medir el nivel o la intensidad de ese engagement en base a los atributos implicados en cada caso en concreto. Para ello, como muestra la Tabla 1, se propone un conjunto de criterios y métricas para el análisis de estos aspectos.

TABLA 1. MEDIDAS DE ATRIBUTOS DEL ENGAGEMENT Y HERRAMIENTAS DE PARTICIPACIÓN CIUDADANA

\begin{tabular}{|c|c|c|c|c|}
\hline \multirow{2}{*}{$\begin{array}{l}\text { Medidas } \\
\text { subjetivas }\end{array}$} & Instrumentos cualitativos & \multicolumn{3}{|c|}{ - Entrevista personal } \\
\hline & Instrumentos cuantitativos & \multicolumn{3}{|c|}{ - Cuestionario (post-experiencia) } \\
\hline \multirow[t]{5}{*}{$\begin{array}{l}\text { Medidas } \\
\text { objetivas }\end{array}$} & $\begin{array}{l}\text { Medidas en herramientas } \\
\text { físicas }\end{array}$ & \multicolumn{3}{|c|}{$\begin{array}{l}\text { - Registros de asistencia presencial } \\
\text { - Número de intervenciones }\end{array}$} \\
\hline & $\begin{array}{l}\text { Medidas en herramientas } \\
\text { virtuales (en cierto periodo } \\
\text { de tiempo) }\end{array}$ & $\begin{array}{l}\text { Medidas de } \\
\text { uso de la } \\
\text { herramienta } \\
\text { a nivel de } \\
\text { ciudadano }\end{array}$ & $\begin{array}{l}\text { Actividad del } \\
\text { usuario }\end{array}$ & $\begin{array}{l}\text { - Número medio de visitas a pági- } \\
\text { na por sesión (acceso) del ciu- } \\
\text { dadano. } \\
\text { - Tiempo medio de una sesión del } \\
\text { ciudadano. }\end{array}$ \\
\hline & & & $\begin{array}{l}\text { Fidelidad del } \\
\text { usuario }\end{array}$ & $\begin{array}{l}\text { - Número de días de acceso del } \\
\text { ciudadano. } \\
\text { - Número de accesos del ciuda- } \\
\text { dano. } \\
\text { - Tiempo empleado en la herra- } \\
\text { mienta por el ciudadano. }\end{array}$ \\
\hline & & $\begin{array}{l}\text { Medidas de } \\
\text { uso de la } \\
\text { herramienta } \\
\text { a nivel de } \\
\text { comunidad }\end{array}$ & $\begin{array}{l}\text { Popularidad de } \\
\text { la herramienta }\end{array}$ & $\begin{array}{l}\text { - Número de ciudadanos partici- } \\
\text { pantes. } \\
\text { - Número de sesiones a la herra- } \\
\text { mienta. } \\
\text { - Número de visitas a página en la } \\
\text { herramienta. }\end{array}$ \\
\hline & & & $\begin{array}{l}\text { Actividad } \\
\text { media de la } \\
\text { comunidad }\end{array}$ & $\begin{array}{l}\text { - Número medio de visitas a pági- } \\
\text { na por sesión. } \\
\text { - Tiempo medio de una sesión. }\end{array}$ \\
\hline
\end{tabular}




\begin{tabular}{|c|c|c|c|c|}
\hline & & & $\begin{array}{l}\text { Fidelidad } \\
\text { media de la } \\
\text { comunidad }\end{array}$ & $\begin{array}{l}\text { - Número medio de días de acce- } \\
\text { so por ciudadano. } \\
\text { - Número medio de accesos por } \\
\text { ciudadano. } \\
\text { Tiempo medio empleado en la } \\
\text { herramienta por ciudadano. }\end{array}$ \\
\hline & & $\begin{array}{l}\text { Medidas } \\
\text { fisiológicas }\end{array}$ & $\begin{array}{l}\text { Análisis de } \\
\text { constantes } \\
\text { biológicas } \\
\text { y respuesta } \\
\text { física }\end{array}$ & $\begin{array}{l}\text { - Respuestas corporales frente a } \\
\text { estímulos exteriores. Ejemplos: } \\
\text { pulsaciones del corazón, parpa- } \\
\text { deo ocular, presión sanguínea. } \\
\text { - Respuestas corporales depen- } \\
\text { dientes de emociones. }\end{array}$ \\
\hline
\end{tabular}

Fuente: Elaboración propia.

En primer lugar, el engagement puede ser medido tanto a nivel individual como a nivel grupal o de colectivo (Salanova y Schaufeli, 2004). Por otra parte, las medidas para analizar el engagement en los sujetos pueden ser subjetivas y objetivas.

Las Medidas Subjetivas son aquellas que pretenden analizar rasgos o aspectos de la psicología del sujeto. Esto se consigue debido a que es el propio sujeto el que describe su experiencia a posteriori a través de una serie de preguntas. Estas métricas van a ayudar a aproximarse a cuestiones como cuáles son los atributos del engagement que entraron en juego durante la actividad de la participación, la intensidad con la que lo hicieron y en qué fase del ciclo del engagement sucedió. Las medidas subjetivas permiten por tanto identificar en el sujeto los atributos del engagement tales como el sentirse parte de un grupo, sentirse eficaz, etc. Estas medidas subjetivas utilizan las siguientes herramientas de análisis:

Instrumentos cualitativos: La herramienta más apropiada dentro de las cualitativas para medir el nivel de engagement es la entrevista personal. La entrevista permite preguntar cuestiones como las razones iniciales del individuo para participar, el grado de involucración, las percepciones del individuo sobre el procedimiento y cuestiones acerca de su nivel de engagement (Robinson et al., 2016). El ciudadano puede expresar mediante una entrevista sus emociones con respecto a la experiencia participativa como por ejemplo si se sintió molesto, frustrado, estimulado, confuso, o sintió que tenía el control en todo momento.

Instrumentos cuantitativos: O'Brien y Toms (2008), Salanova y Schaufeli (2004) indican que el cuestionario es el instrumento más apropiado para «recolectar» el nivel de engagement de los usuarios. Mediante el cuestionario se diseñan preguntas con el objetivo de cuantificar o clasificar a los individuos en base a sus respuestas. Permite preguntar cuestiones como la frecuencia con la que los individuos llegan al nivel de engagement a través de la utilización de distintas herramientas (Robinson et al., 2016). Otro análisis posible es cruzar datos sobre los niveles de participación con datos sobre la intensidad del engagement o con las herramientas utilizadas para participar.

Las Medidas Objetivas pueden medir de forma más adecuada elementos relacionados con las propias herramientas de participación utilizadas en los distintos niveles de participación (niveles de Información, Consulta y Delegación de poder en el ciudadano). Estas herramientas permiten capturar datos de forma objetiva sobre los cambios y la evolución del engagement a lo largo del tiempo. Se distinguen aquí aquellas métricas que pueden aplicarse en las herramientas físicas de participación de las aplicadas en herramientas virtuales.

Medidas en las herramientas físicas: Memarovic et al. (2012) señalan que herramientas como las Pantallas Públicas Interactivas tienen la función y capacidad de registrar la actividad que se realiza en ellas. Pueden obtenerse aquí tanto registros de asistencia presencial como el número de intervenciones que realizan los ciudadanos en el dispositivo. En herramientas como las plataformas físicas o los Living labs pueden medirse aspectos como la asistencia a reuniones, el grado de intervención en proyectos conjuntos, etc.

Medidas en las herramientas virtuales: permiten medir distintos aspectos que suceden en un momento concreto en el tiempo. Pueden distinguirse aquí distintos tipos de métricas:

- Medidas sobre el uso de la herramienta de participación por parte del ciudadano: permiten medir por un lado la actividad del ciudadano (es decir, el número de visitas a una página por sesión y tiempo medio que permanece el usuario en ese sistema informático). Además, puede medirse lo 
que en Ciencias de la Computación se denomina fidelidad (que contempla el número de días que el ciudadano ha utilizado esa herramienta, el número de accesos a la misma y el tiempo empleado).

- Medidas sobre el uso de la herramienta de participación por parte de un colectivo o comunidad: en cuanto a las métricas que pueden aplicarse a un colectivo o comunidad para medir el engagement en participación puede utilizarse, en primer lugar, la popularidad de la herramienta (referida al número de participantes que utilizan esa herramienta). En segundo lugar, puede medirse la actividad media del colectivo en esa herramienta de participación (mediante la medición del tiempo medio de utilización de la herramienta o el tiempo medio de cada sesión). Por último, puede medirse la fidelidad media de la comunidad (controlando aspectos como la media de días de acceso o el tiempo medio empleado en la misma).

- Medidas fisiológicas: Distintos autores en la literatura han tratado de medir el engagement a través de la respuesta física provocada por la actividad a través de distintos sensores conectados al cuerpo humano del individuo (Jennett et al., 2008). Respuestas como la fatiga, la atención o la actividad mental pueden medirse con sensores oculares. El estrés puede medirse por la presión respiratoria. Otras emociones se corresponden a respuestas fisiológicas como la presión sanguínea. Este tipo de análisis pueden realizarse a lo largo de la actividad participativa del individuo.

En la literatura se ha propuesto medir el engagement mediante técnicas distintas (Attfield et al., 2011; Chapman, 1997; Jacques, 1996; Konradt y Sultz, 2001; Lehmann et al., 2012; O'Brien y Toms, 2008; Robinson et al., 2016; Webster y Ho, 1997), sin embargo una minoría de estos estudios han sido validados (Attfield et al., 2011). Según O' Brien y Toms (2008) existen dificultades a la hora de medir el engagement debido a la complejidad de conectar las métricas al engagement. Elegir sólo alguna de estas métricas no ofrecería un análisis correcto del engagement en un ciudadano. En cambio, la combinación de distintas herramientas y métricas subsanaría errores o vacíos que pueda tener cada métrica por separado. El análisis de los datos recogidos podría proporcionar información interesante como la cantidad de personas que experimentan engagement o realizar comparaciones entre individuos y en distintos momentos temporales con el objetivo de medir los niveles de engagement en cada fase participativa (Salanova y Schaufeli, 2004). Además, puede ofrecer una visión de población segmentada por género y por edad que va a aportar información sobre los tipos de público objetivo a los que dirigir los esfuerzos por potenciar el engagement a través de la utilización de distintas herramientas en cada caso.

\section{CONCLUSIONES Y TRABAJO FUTURO}

En este artículo, el engagement se ha definido como un estado psicológico en el que el individuo disfruta y encuentra satisfacción en la actividad que desempeña, por lo que tiende a repetir ésta. Durante el estudio descriptivo realizado se han obtenido una serie de atributos que pertenecen de forma intrínseca al sujeto y otros, que por el contrario, pertenecen al sistema que se emplea. Gracias a la identificación de las herramientas de participación que pueden utilizarse en la ciudad inteligente, puede plantearse el diseño de las mismas con el objetivo de cuidar distintos aspectos que fomenten los atributos del engagement. Estas herramientas no sólo reducirían costes al ciudadano de corte económico, organizativo y temporal (Colombo, 2006), sino que podrían ser susceptibles de mejoras para potenciar en el individuo un estado psicológico de engagement que incrementase los niveles de participación ciudadana a escala individual y social.

Como trabajo futuro es necesario un estudio explicativo que valide el modelo propuesto mediante la realización de distintos análisis que muestren, por un lado, si existe relación entre las herramientas de participación ciudadana y su influencia en la predisposición del ciudadano a participar en las iniciativas de las ciudades inteligentes y, por otro lado, el grado en el que se produce esta influencia.

No obstante, además de cuidar distintos aspectos en las herramientas de participación, deben tenerse en cuenta medidas de carácter político como la mejora del acceso a internet o distintas formas de empoderamiento que popularicen la utilización de las distintas herramientas de participación y eliminen las barreras de participación al ciudadano (Mandarano et al., 2010; Ramilo Araujo y Fernández Rupérez, 2012). Además, debe prestarse atención a cuestiones como el papel del ciudadano dentro de las iniciativas de ciudades inteligentes. Concretamente a cómo influirían una mayor delegación de poder y competencias en el individuo en distintos atributos del engagement como la autoestima, el reto o la independencia. 


\section{REFERENCIAS BIBLIOGRÁFICAS}

ABOULAFIA, A., y BANNON, L. J. (2004). "Understanding affect in design: An outline conceptual framework". Theoretical Issues in Ergonomic Science, 5 (1), 4-15. https://doi.org/10.1080/1463922031000086708.

ALAWADHI, S., ALDAMA-NALDA, A., CHOURABI, H., GIL-GARCIA, J. R., LEUNG, S., MELLOULI, S. y WALKER, S. (2012). "Building understanding of smart city initiatives". In 11th International Conference on Electronic Government, págs. 40-53. https://doi.org/10.1007/978-3-642-33489-4_4.

ALBINO, V., BERARDI, U., y DANGELICO, R. M. (2015). "Smart cities: Definitions, dimensions, performance, and initiatives". Journal of Urban Technology, 22 (1), 3-21. https://doi.org/10.1080/10630732.2014.942092.

ANDRÉS, J., y BONIVENTO, H. (2009). "Problemas institucionales de la participación ciudadana: análisis conceptual y aplicación al caso colombiano". XXII Concurso del CLAD Sobre Reforma del Estado y Modernización de la Administración Pública, págs. 1-23.

ANTTIROIKO, A. V. (2016). "City-as-a-Platform: The rise of participatory innovation platforms in Finnish cities". Sustainability, 8 (9), 922. https://doi.org/10.3390/su8090922.

ATTFIELD, S., KAZAI, G., LALMAS, M. y PIWOWARSKI, B. (2011). "Towards a science of user engagement" (position paper), en WSDM 2011 workshop on user modelling for Web applications, págs. 9-12.

AVERSANO, P., RAJU, A., MECHANT, P. y BALLON, P. (2013). "Reuse potential assessment framework for gamification-based smart city pilots", en 2nd International Biennial Conference Hybrid City, Subtle Revolutions, págs. 17-23.

BANDURA, A. (2001). "Social cognitive theory: An agentic perspective". Annual review of psychology, 52 (1), 1-26. https://doi.org/10.1146/annurev.psych.52.1.1.

BONSÓN, E., ROYO, S. y RATKAI, M. (2015). 'Citizens' engagement on local governments' Facebook sites: An empirical analysis: The impact of different media and content types in western Europe". Government Information Quarterly, 32 (1), 52-62. https://doi.org/10.1016/j.giq.2014.11.001.

BONSÓN, E., TORRES, L., ROYO, S. y FLORES, F. (2012). "Local e-government 2.0: Social media and corporate transparency in municipalities". Government information quarterly, 29 (2), 123-132.

BOVAIRD, T. (2007). "Beyond engagement and participation: User and community coproduction of public services". Public Administration Review, 67 (5), 846-860. https://doi.org/10.1111/j.1540-6210.2007.00773.x.

BRUGUÉ, Q., FONT, J. y GOMÀ, R. (2003). Participación y democracia. Asociaciones y poder local. Movimientos sociales: cambio social y participación. Madrid: UNED, 109-132.

BRYNSKOV, M., BERMÚDEZ, J. C. C., FERNÁNDEZ, M., KORSGAARD, H., MULDER, I., PISKOREK, K. ... y de WAAL, M. (2014). "Urban interaction design: Towards city making". Urban IxD Booksprint, 96.

BRYER, T. A. (2013). "Designing social media strategies for effective citizen engagement: A case example and a model". National Civic Review, 102 (1), 43-50. https://doi.org/10.1002/ncr.21114.

BUDGE, I. (1996) The New Challenge of Direct Democracy. Londres: Polity Press.

BULL, R. y AZENOUD, M. (2016) "Smart participation - social learning: a model of participation". Energy Proceedings of ICE.

BUTTERISS, C., CROZIER, M., SCANLAN, R. y GOBEY, T. (2012). Online Consultation Guide Book: A practice guide for EngagementHQ. http://demonstration.engagementhq.com/2636/documents/24399.

CARR, S. (1992). Public space. Cambridge University Press.

CASTELLS, M. (2000). Information age: Economy, society and culture. Volume 1: The rise of the network society. Blackwell Publishers.

CASTELNOVO, W., MISURACA, G. y SAVOLDELLI, A. (2015). "Citizen's engagement and value co-production in smart and sustainable cities", en 2015 International Conference on Public Policy, págs. 1-16.

CHAPMAN, P. M. (1997). Models of engagement: Intrinsically motivated interaction with multimedia learning software. Doctoral dissertation, University of Waterloo.

CHOURABI, H., NAM, T., WALKER, S., GIL-GARCIA, J. R., MELLOULI, S., NAHON, K., SCHOLL, H. J. (2011). "Understanding smart cities: An integrative framework", en 2011 Annual Hawaii International Conference on System Sciences. https://doi.org/10.1109/hicss.2012.615.

COLOMBO, C. (2006). "Innovación democrática y TIC, ¿hacia una democracia participativa?”. Revista de Internet, Derecho y Política, 3, 1.699-8.154.

CROSS, J. (2013). "Three Myths of Behavior Change: What You Think You Know That You Don't”. TEDxCSU. 20 March.

DALSGAARD, P. y ERIKSSON, E. (2013). "Large-scale participation: a case study of a participatory approach to developing a new public library", en 2013 SIGCHI Conference on Human Factors in Computing Systems, págs. 399-408.

EUROPEAN PARLIAMENT (2014). Mapping Smart Cities in the EU.

EUROPEAN UNION (2006). The Helsinki Manifesto.

DíAZ, A. (2017). "Participación ciudadana en la gestión y en las políticas públicas". Gestion y Politica Publica, 26 (2), 341-379. 
GAPP. Nueva Época - N. 19, mayo 2018 - ISSN: 1989-8991 - DOI: 10.24965/gapp.v0i19.10505 - [Págs. 50-69]

Engagement en ciudades inteligentes. Diseño de un marco de análisis teórico y aplicado para la participación ciudadana María E. Cortés-Cediel / Olga Gil

FLORIDA, R. (2003). "Cities and the Creative Class". City \& Community, 2 (March), 3-19. https://doi.org/10.1111/15406040.00034.

FORRESTER, J. P. y WATSON, S. S. (1994). "An assessment of public administration journals: The perspective of editors and editorial boards members". Public Administration Review, 54 (5), 474-482. https://doi. org/10.2307/976433.

FRIERIKSON, B. L. (2002). Positive emotions. Handbook of Occupational Health Psychology, 19-33.

GANUZA FERNÁNDEZ, E. (2008). “¿A qué llamamos participar en democracia? Diferencias y similitudes en las formas de participación”. Revista Internacional de Sociología, 66, 89-113.

GIFFINGER, R. (2007). "Smart cities Ranking of European medium-sized cities", 13-18.

GIL, O., NAVÍO, J. y DE HEREDIA, M. P. (2016). “¿Cómo se gobiernan las ciudades?”. TAG-Revista institucional del Collegi d'Aparelladors, Arquitectes Tècnics i Enginyers d'Edificació de Tarragona (76), 38-39.

GROUP, W. B. (2016). Evaluating digital citizen engagement: A practical guide. World Bank, Washington, DC.

HOWE, J. (2006). "The rise of crowdsourcing". Wired magazine, 14 (6), 1-4.

INGLEHART, R. (1991). El cambio cultural en las sociedades industriales avanzadas (núm. 121). CIS.

JACQUES, R. D. (1996). The nature of engagement and its role in hypermedia evaluation and design. Thesis, South Bank University.

JENNETT, C., COX, A. L., CAIRNS, P., DHOPAREE, S., EPPS, A., TIJS, T. y WALTON, A. (2008). "Measuring and defining the experience of immersion in games". International journal of human-computer studies, 66 (9), $641-661$. https://doi.org/10.1016/j.ijhcs.2008.04.004.

JENNINGS, M. (2000). "Theory and models for creating engaging and immersive ecommerce Web sites", en 2000 ACM SIGCPR Conference on Computer Personnel Research, págs. 77-85. https://doi.org/10.1145/333334.333358.

KAASE, M. y NEWTON, K. (1995). Beliefs in government (vol. 5). OUP Oxford. $h$ ttps://doi.org/10.1093/0198294727.001.0001.

KONRADT, U. y SULZ, K. (2001). "The experience of flow in interacting with a hypermedia learning environment". Journal of Educational Multimedia and Hypermedia, 10 (1), 69-84.

KUKKA, H.,HOSIO, S. y GONCALVES, J. (2014). "Situated engagement and virtual services in a smart city", en 2014 IEEE 7th International Conference on Service-Oriented Computing and Applications, págs. 328-331.

LEV-ON, A. y STEINFELD, N. (2015). "Local engagement online: Municipal Facebook pages as hubs of interaction". Government Information Quarterly, 32 (3), 299-307. https://doi.org/10.1016/j.giq.2015.05.007.

LEHMANN, J., LALMAS, M. YOM-TOV, E. y DUPRET, G. (2012). Models of user engagement. User Modeling, Adaptation, and Personalization, 164-175. https://doi.org/10.1007/978-3-642-31454-4_14.

MANDARANO, L., MEENAR, M. y STEINS, C. (2010). "Building social capital in the digital age of civic engagement". Journal of Planning Literature, 25 (2), 123-135. https://doi.org/10.1177/0885412210394102.

MASLOW, A. H. (1954). Motivación y personalidad. Madrid: Díaz de Santos S. A.

MARTINS, S. (2002). "Novos instrumentos de participaçao: entre a participaçao e a deliberaçao", en MOREIRA DA SILVA, E. y SCHETTINI MARTINS, E. (eds.), 1-21.

MATLIN, M. W. (1994) Cognition (3rd ed.), Dallas, TX: Harcourt Brace.

MEIJER, A. (2016). "Coproduction as a structural transformation of the public sector". International Journal of Public Sector Management, 29 (6), 596-611. https://doi.org/10.1108/ijpsm-01-2016-0001.

MEIJER, A. y BOLIVAR, M. P. R. (2015). "Governing the smart city: a review of the literature on smart urban governance". International Review of Administrative Sciences. https://doi.org/10.1177/0020852314564308.

MEMAROVIC, N., LANGHEINRICH, M., ALT, F., ELHART, I., HOSIO, S. y RUBEGNI, E. (2012, November). "Using public displays to stimulate passive engagement, active engagement, and discovery in public spaces", en 4th Media Architecture Biennale Conference: Participation, págs. 55-64.

MERGEL, I. (2013). "A framework for interpreting social media interactions in the public sector". Government Information Quarterly, 30 (4), 327-334. https://doi.org/10.1016/j.giq.2013.05.015.

MEZIROW, J. (1994). "Understanding transformation theory". Adult education quarterly, 44 (4), 222-232. https://doi. org/10.1177/074171369404400403.

NAHL, D. y BILAL, D. (Eds.). (2007). Information and emotion: The emergent affective paradigm in information behavior research and theory. Information Today.

NAM, T. y PARDO, T. A. (2011). "Conceptualizing smart city with dimensions of technology, people, and institutions", en 12th Annual International Digital Government Research Conference on Digital Government. https://doi. org/10.1145/2037556.2037602.

NIEDERER, S. y PRIESTER, R. (2016). "Smart citizens: Exploring the tools of the urban bottom-up movement". Computer Supported Cooperative Work, 25 (2-3), 137-152.

NORRIS, D. F. y REDDICK, C. G. (2013). "Local e-government in the United States: Transformation or incremental change?". Public Administration Review, 73 (1), 165-175.

O'BRIEN, H. L. y TOMS, E. G. (2008). "What is user engagement? A conceptual framework for defining user engagement with technology". Journal of the Association for Information Science and Technology, 59 (6), 938-955.

OPROMOLLA, A., VOLPI, V., INGROSSO, A. y MEDAGLIA, C. M. (2015). "Co-design practice in a smart city context through the gamification approach: A survey about the most suitable applications", en 2015 International Conference on Distributed, Ambient, and Pervasive Interactions, págs. 578-589. 
ORGANISATION FOR ECONOMIC CO-OPERATION AND DEVELOPMENT (2001). Citizens as partners Information, Consultation and Public Participation in Policy-Making, pág. 23. Paris: OECD.

ORGANISATION FOR ECONOMIC CO-OPERATION AND DEVELOPMENT (2003). Promise and problems of e-democracy: Challenges of online citizen engagement. Paris: OECD.

PAULOS, E., HONICKY, R. y HOOKER, B. (2008). Citizen science: Enabling participatory urbanism. Urban Informatics: Community Integration and Implementation.

PAULOS, E. y JENKINS, T. (2005). "Urban probes: encountering our emerging urban atmospheres", en 2005 SIGCHI Conference on Human factors in computing systems. págs. 341-350. https://doi.org/10.1145/1054972.1055020.

PHAM, L. y LINEHAN, C. (2016). "Crowdsourcing: Tackling challenges in the engagement of citizens with smart city initiatives", en SEACHI 2016 Conference on Smart Cities for Better Living with HCI and UX, págs. 28-31. https:// doi.org/10.1145/2898365.2899799.

RAMILO ARAUJO, C. y FERNÁNDEZ-RUPÉREZ, R. (2012): "Ciudadanía y participación en la Sociedad de la Información y del Conocimiento", GIGAPP Estudios Working Papers, 21: 1-27.

REDDEL, T. y WOOLCOCK, G. (2004). "From consultation to participatory governance? A critical review of citizen engagement strategies in Queensland". Australian Journal of Public Administration, 63 (3), 75-87.

ROBBINS, T. (2007). Why We Do What We Do. TED Talks. 16th January 2016.

ROBINSON, L., PHILLIPS, J., BRETT, E., DIGITAL, T. H. E. y SQUARE, P. (2016). What Motivates Citizens to Participate? DIGITAL.

RODRÍGUEZ-BOLÍVAR, M. P. y MEIJER, A. (2015). "Smart governance. Using a literature review and empirical analysis to build a research model". Social Science Computer Review, 34 (6), 673-692. https://doi. org/10.1177/0894439315611088.

SALANOVA, M., LLORENS, S. y GARCÍA-RENEDO, M. (2003). “¿Por qué se están quemando los profesores?” Prevención, trabajo y salud, 28, 16-20.

SALANOVA SORIA, M. y SCHAUFELI, W. B. (2004). "El engagement de los empleados: un reto emergente para la dirección de los recursos humanos". Revista de trabajo y Seguridad Social. Recursos humanos, (261), 109-138.

SALIM, F. y HAQUE, U. (2015). "Urban computing in the wild: A survey on large scale participation and citizen engagement with ubiquitous computing, cyber physical systems, and Internet of Things". International Journal of Human-Computer Studies, 81, 31-48. https://doi.org/10.1016/j.ijhcs.2015.03.003.

SCHROETER, R. (2012). "Engaging new digital locals with interactive urban screens to collaboratively improve the city", en 2012 ACM Conference on Computer Supported Cooperative Work, págs. 227-236. https://doi. org/10.1145/2145204.2145239.

SIEBER, R. (2006). "Public participation geographic information systems: A literature review and framework". AAG Annals, 96 (3), 491-507. https://doi.org/10.1111/j.1467-8306.2006.00702.x.

SPOTTKE, J. (2006). Ethnographic Reflection on Group Formation in Blizzard's World of Warcraft. University of Central Florida Thesis.

STONE, D. N., DECI, E. L. y RYAN, R. M. (2005). "Beyond talk: Creating autonomous motivation through selfdetermination theory". Journal of General Management, 34 (3), 75-91.

SUBIRATS, J. (2013). "Internet y participación política: ¿nueva política?, ¿nuevos actores?”. Revista de Ciencias Sociales, 26 (33), 55-72.

UNITED NATIONS. DEPARTMENT OF ECONOMIC (2008). World Economic Situation and Prospects 2008. United Nations Publications. https://doi.org/10.18356/a9d21eef-en.

WEBSTER, J. y AHUJA, J. S. (2004). Enhancing the design of Web navigation systems: The influence of user disorientation on engagement and performance. Unpublished manuscript.

WEBSTER, J. y HO, H. (1997). "Audience engagement in multimedia presentations". Sigmis Database, 28 (2), $63-77$. https://doi.org/10.1145/264701.264706.

WHEELAN, S. (2005). The Handbook of Group Research and Practice. Sage Publishing.

WHYTE, W. H. (1980). The social life of small urban spaces.

YANKOVIC, B. (2011). Emociones, sentimientos, afecto. El desarrollo emocional. 